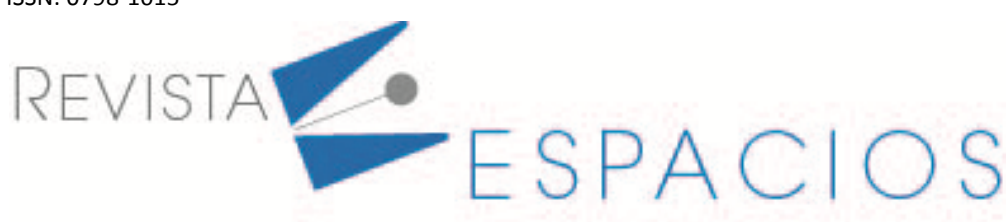

\title{
Inteligencia emocional y rendimiento académico en universitarios: una revisión sistemática
}

\section{Emotional intelligence and academic performance in university students: a systematic review}

\author{
RODRÍGUEZ RODRÍGUEZ, Juan C. ${ }^{1}$ \\ RODRÍGUEZ RODRÍGUEZ, José A. ${ }^{2}$ \\ RODRÍGUEZ GÓNGORA, Josefina ${ }^{3}$ \\ BOJÓRQUEZ DÍAZ, Cecilia I. ${ }^{4}$
}

\begin{abstract}
Resumen
La Inteligencia Emocional es capaz de predecir de forma más significativa el éxito personal. Inteligencia emocional (IE) y rendimiento académico (RA) han sido estudiadas por separado de forma pormenorizada, pero ¿cómo se relacionan? En este estudio se recoge una revisión sistemática de tres artículos en castellano que abarcan la relación entre las variables IE y RA en universitarios. Los resultados del análisis indican que existe una correlación significativa entre ambos y que niveles altos de IE predeciría un elevado RA.

Palabras clave: inteligencia emocional, rendimiento académico, universitarios

Abstract

Emotional Intelligence can predict personal success in a more meaningful way. Emotional intelligence (EI) and academic performance (AP) have been studied separately in detail, but how are they related? This study includes a systematic review of three articles in spanish that covers the relationship between the variables El and AR in university students. The results of the analysis indicate that there is a significant correlation between the two and that high levels of El would predict a high AR.

key words: emotional intelligence, academic performance, university students
\end{abstract}

\section{Introducción}

La Inteligencia Emocional (IE) es un concepto que en los últimos años ha tenido gran repercusión científica. Hasta hace unos años, en el mundo científico-académico prevalecía el concepto de inteligencia general como capacidad de adquirir y transmitir información, lo que parecía la causa de partida del éxito personal. Moreno, Vicente, \& Martínez (1998) y Salovey \& Mayer (1990) crearon el concepto de Inteligencia Emocional, que en un principio tuvo poco reconocimiento hasta que Goleman en 1996 publicó su libro titulado Inteligencia Emocional. Allí en

\footnotetext{
${ }^{1}$ Instituto de Investigación en Ciencias Sociales y Educación. Universidad de Atacama. Chile. HUM-1028. Investigación Internacional Comparada. Universidad de Almería. España. jucar61@ual.es

2 Universidad de Almería. España.jrr962@inlumine.ual.es

3 Instituto de Investigación en Ciencias Sociales y Educación. Universidad de Atacama. Chile. HUM-1028. Investigación Internacional Comparada. Universidad de Almería. España.jrg293@inlumine.ual.es

${ }^{4}$ Instituto Tecnológico de Sonora. México. cecilia.bojorquez@itson.edu.mx
} 
adelante ya no se trataba puramente de lo cognitivo, sino también del plano emocional, se trataba de reconocer y gestionar las propias emociones y las de los demás. Desde entonces, se abrieron multitud de líneas de investigación y entrenamiento emocional en diferentes campos: empresarial (Caruso \& Salovey, 2005), educación (Ilaja \& Reyes, 2016) o salud (Sánchez-López, Megías, Gómez, Gutiérrez, \& Fernández-Berrocal, 2018).

Son muchos, y cada vez más, los estudios que demuestran como altos niveles de inteligencia emocional predicen buenas habilidades sociales (Rodríguez F. , 2017), rendimiento académico (Del Rosal, Moreno-Manso, \& Bermejo, 2016), buen ejercicio laboral (Apaza, 2018), bienestar (Cejudo, López-Delgado, \& Rubio, 2016) y buena calidad de vida (Luque-Reca, 2015)

Cuando se habla de éxito profesional, se tiende a asociarlo a variables académicas como el rendimiento académico. Algunos estudios apuntan a que la inteligencia emocional es un mejor predictor del éxito profesional y personal (Marín, Infante, \& Troyano, 2000). Tanto el Rendimiento Académico como la Inteligencia Emocional son variables que parecen apuntar en la misma dirección, según diferentes estudios, pero ¿cuál es la relación entre ambas?

Fernández-Berrocal \& Ruiz-Aranda (2008) estudiaron cómo la Inteligencia emocional y el rendimiento académico se correlacionan con el bienestar psicológico, las relaciones interpersonales y la aparición de conductas disruptivas. Los estudiantes que participaron en su investigación, cuya inteligencia emocional fue clasificada de bajo nivel, se mostraron propensos a delinquir, presentaron síntomas depresivos y ansiosos y la calidad de sus relaciones fue significativamente baja. Por su parte, Ferragut y Fierro (2012) realizaron un estudio en adolescentes en el que concluyeron que ambas variables, inteligencia emocional y rendimiento académico, correlacionaban de forma significativa con el bienestar psicológico. Aquellos alumnos cuya inteligencia emocional se situaba por encima de la media y cuyo expediente también estaba por encima del promedio, puntuaban mejor en la escala de bienestar psicológico.

Buenrostro-Guerrero et al. (2012) observaron en 439 adolescentes que aquellos cuya inteligencia emocional era más alta poseían mayores puntajes promedio académicos. Esta relación ha sido estudiada de forma múltiple en los últimos años, donde encontramos gran variedad de estudios que la estudian y la conjugan con otras variables como resiliencia o habilidades sociales. De la misma forma, esta relación ha sido estudiada en distintas poblaciones: población infantil (Acosta \& Clavero, 2017), preadolescentes (Valenzuela-Santoyo \& Portillo, 2018), universitarios (Pérez \& Castejón, 2007), profesores (Jiménez \& López-Zafra, 2013) e incluso en personas diagnosticadas con Trastorno del Espectro Autista (Núñez, 2018).

Por tanto, existen numerosas publicaciones que enlazan estos conceptos por separado y otras que intentan unirlos a nuevas variables. Aun así, en los últimos años son pocos los estudios que se centran en la relación pura de ambas variables. Por ello, se hace necesario profundizar en esta relación acotando y reduciendo esta revisión sistemática hacia aquellos trabajos que correlacionen inteligencia emocional y rendimiento académico en universitarios.

\section{Metodología}

\section{Procedimiento de búsqueda}

Para lograr una buena estructura de este manuscrito, los pasos que se han seguido para establecer esta revisión sistemática se recogen en los estándares sobre la realización de una revisión sistemática en ciencias de la salud de Perestelo-Pérez (2013).

La búsqueda bibliográfica se ha realizado en tres bases de datos: Psyclnfo, Psicodoc y Dialnet. Esta búsqueda se llevó a cabo durante los meses noviembre y diciembre de 2020, prestando una mayor atención a aquellos 
artículos cuyo impacto científico ha sido mayor. Asimismo, la búsqueda limitó a los últimos cinco años, desde 2015 hasta 2020. Las palabras clave que se usaron para la búsqueda fueron "Emotional Intelligence", "academic performance" y "university students"; lo que sería igualmente "Inteligencia Emocional", "rendimiento académico" y "estudiantes universitarios" en el caso de los artículos titulados en castellano.

\section{Criterios de inclusión y exclusión}

Como criterios de inclusión para la selección de los trabajos se tuvo en cuenta: (a) que los participantes fueran estudiantes universitarios independientemente de su edad y sexo; (b) que los estudios fueran empíricos; (c) que contaran con instrumentos validados estadísticamente; (d) que estuvieran publicados en los últimos diez años; y (e) que solamente se centraran en medir inteligencia emocional y rendimiento académico sin añadir otra clase de variables

Como criterios de exclusión, se omitieron aquellos estudios que estuvieran escritos en un idioma distinto al castellano. También fueron descartados los estudios que no incluían datos relevantes como el número de participantes o fundamentos sobre la fiabilidad de los instrumentos, estudios cuyo carácter fuera de tipo cualitativo en lugar de cuantitativo y escritos diferentes a un artículo científico como actas de congresos o ensayos breves. Por último, se descartaron aquellos artículos que no fueron revisados por pares.

\section{Selección de los estudios}

Primeramente, se realizó una lectura del título y del resumen de los trabajos que habían sido seleccionados al cumplir los criterios de búsqueda que se han marcado. Posteriormente, se llevó a cabo una lectura comprensiva de los artículos y se examinaron recogiendo información del título, autores, año de publicación, características de la muestra e instrumentos.

\section{Codificación de los resultados}

A partir de lo expuesto y tras considerar los criterios, se llevó a cabo la extracción de los datos diseñando el manual de codificación (véase Tabla 1) a través de una hoja de cálculo Excel en la que se ordenaron las características más importantes: Autoría, año de publicación, objetivo, muestra, variables, instrumento, lugar y resultados. En el caso de instrumentos, en los estudios siempre se ha medido el rendimiento académico por la media del expediente académico, por lo que simplemente se tuvo en cuenta qué cuestionario o inventario se usó para medir la inteligencia emocional. 
Tabla 1

Resumen de la información registrada de cada estudio

\begin{tabular}{|c|c|c|c|c|c|c|c|}
\hline Autoría & Año & Objetivo & Muestra & $\begin{array}{l}\text { Tipo de } \\
\text { muestreo }\end{array}$ & Instrumento IE & Lugar & Resultados \\
\hline Díaz & 2013 & $\begin{array}{l}\text { Determinar el grado } \\
\text { de relación entre la } \\
\text { inteligencia emocional } \\
\text { y el rendimiento } \\
\text { académico } \\
\text { de los estudiantes de } \\
\text { Farmacia y Bioquímica }\end{array}$ & $n=150$ & Incidental & $\begin{array}{l}\text { Inventario } \\
\text { Emocional De } \\
\text { BarOn } \\
\text { Inventario de } \\
\text { Coeficiente } \\
\text { Emocional (I-CE) }\end{array}$ & $\begin{array}{l}\text { Universidad } \\
\text { Los Andes (Perú) }\end{array}$ & $\begin{array}{l}\text { Correlación } \\
\text { significativa entre } \\
\text { inteligencia } \\
\text { emocional y } \\
\text { rendimiento } \\
\text { académico }\end{array}$ \\
\hline $\begin{array}{l}\text { Rodas y } \\
\text { Rojas }\end{array}$ & 2015 & $\begin{array}{l}\text { Determinar } \\
\text { la relación funcional } \\
\text { entre el rendimiento } \\
\text { académico } \\
\text { y los niveles de } \\
\text { inteligencia emocional }\end{array}$ & $n=36$ & Aleatorio & $\begin{array}{l}\text { Se crea y se } \\
\text { valida un } \\
\text { cuestionario de } \\
\text { IE siguiendo las } \\
\text { dimensiones de } \\
\text { Brace (2008) }\end{array}$ & $\begin{array}{l}\text { Universidad } \\
\text { Nacional Pedro } \\
\text { Ruiz Gallo (Perú) }\end{array}$ & $\begin{array}{l}\text { Niveles elevados de } \\
\text { inteligencia } \\
\text { emocional predicen } \\
\text { un alto rendimiento } \\
\text { académico }\end{array}$ \\
\hline $\begin{array}{l}\text { Páez y } \\
\text { Castaño }\end{array}$ & 2015 & $\begin{array}{l}\text { Describir la } \\
\text { inteligencia emocional } \\
\text { y determinar su } \\
\text { relación } \\
\text { con el rendimiento } \\
\text { académico en } \\
\text { estudiantes } \\
\text { universitarios. }\end{array}$ & $n=263$ & $\begin{array}{l}\text { Estratificado } \\
\text { por } \\
\text { programa y } \\
\text { género }\end{array}$ & $\begin{array}{l}\text { Inventario } \\
\text { Emocional De } \\
\text { BarOn }\end{array}$ & $\begin{array}{l}\text { Universidad de } \\
\text { Manizales } \\
\text { (Colombia) }\end{array}$ & $\begin{array}{l}\text { Se encontró } \\
\text { correlación entre } \\
\text { el valor de IE y nota } \\
\text { promedio hasta el } \\
\text { momento ( } p=.019) \text {, } \\
\text { mayor para } \\
\text { Medicina ( } p=.001) \text {, } \\
\text { seguido de } \\
\text { Psicología }(p=.066)\end{array}$ \\
\hline
\end{tabular}

Nota. ${ }^{*}$ IE=inteligencia emocional

\section{Resultados}

La Figura 1 muestra el diagrama de flujo que corresponde al proceso de selección de los documentos. Fueron identificados un total de 48 artículos de revista, de los cuales, debido al uso de tres bases de datos que comparten revistas científicas, se encontraron que cuatro estaban duplicados. Teniendo en cuenta los criterios de inclusión y exclusión, se optó por seleccionar 14, ellos se sacaron tres para esta revisión sistemática debido a que eran los únicos que no analizaban otro tipo de variables.

En primer lugar, Díaz (2013) evaluó la inteligencia emocional en alumnos de Farmacia y Bioquímica de la Universidad Los Andes de Perú y examinó su relación con el rendimiento académico que presentaban en ese momento. Los resultados confirmaron esta correlación y además mostraron que los componentes intrapersonal, interpersonal, adaptabilidad, manejo de estrés y estados de ánimo actúan de forma estadísticamente significativa con el rendimiento académico.

Por su parte, Rodas y Rojas (2015) llevaron a cabo un estudio con la misma finalidad que el anterior, pero llevado a cabo en la Universidad Nacional Pedro Ruiz Gallo (Perú). Usando muestreo probabilístico, reunieron un total de 36 estudiantes de los que concluyeron que la relación entre ambas variables es muy dependiente, siendo así que altos niveles de inteligencia emocional predicen un rendimiento académico óptimo.

Por último, Páez y Castaño (2015) evaluaron la inteligencia emocional con el Inventario Emocional de BarOn a 263 estudiantes y encontraron un cociente de inteligencia emocional promedio de 46,51. Analizando por facultades, los resultados mostraron que los niveles de Inteligencia Emocional, en orden decreciente, serían: Economía, Medicina, Psicología y Derecho. Además, únicamente se encontró relación significativa entre inteligencia emocional y rendimiento Académico en Medicina y Psicología, con resultados más evidentes en el primero. 
Figura 1

Diagrama de flujo de la selección de los estudios

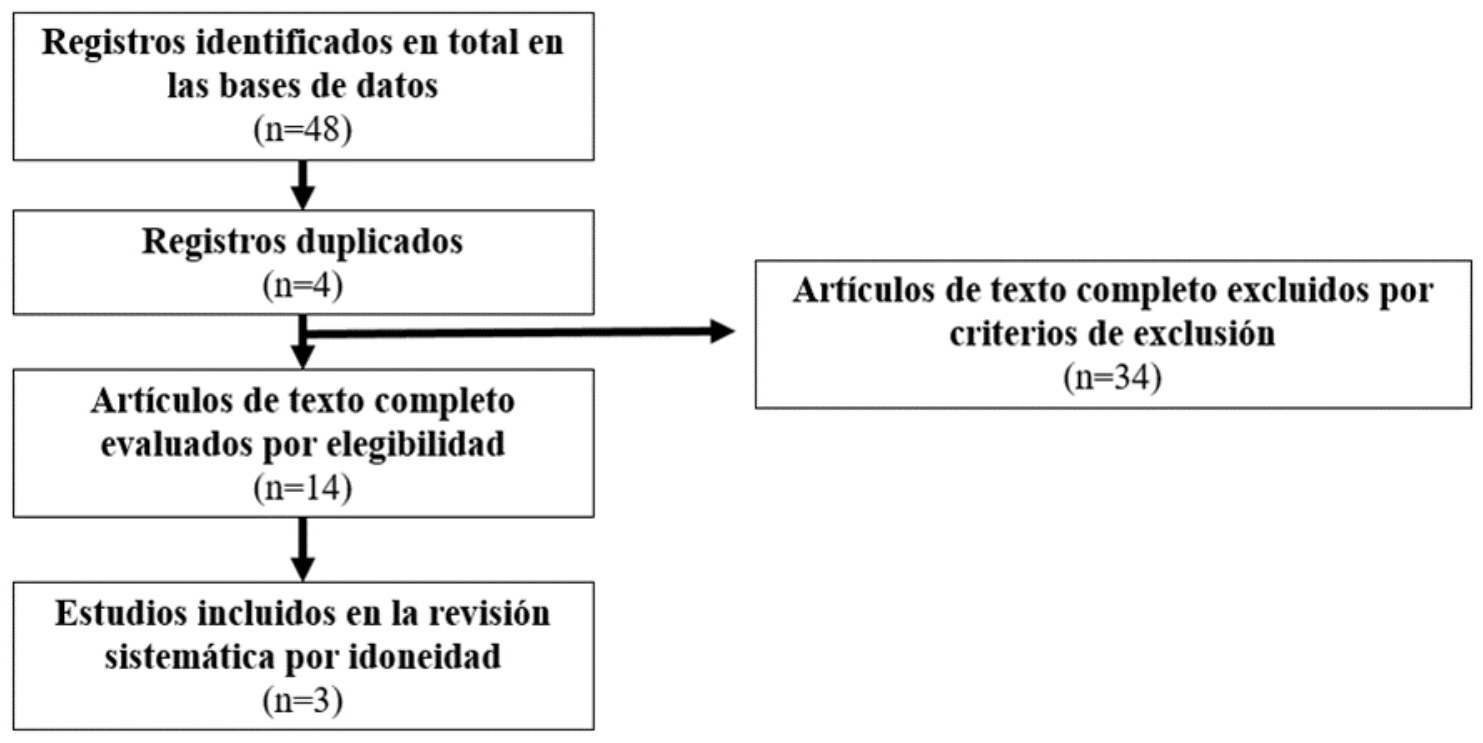

Fuente: Elaboración propia

\section{Discusión}

Inteligencia emocional y rendimiento académico han sido estudiados de forma múltiple en los últimos años, estando ambas íntimamente ligadas a otras variables de corte emocional y cognitivo (Caruso \& Salovey, 2005; Ilaja y Reyes, 2016; Sánchez-López et al., 2018). Aun así, son pocos los estudios en castellano que estudian esta relación de forma exclusiva.

Por ello, el objetivo principal del estudio fue establecer qué relación encontramos entre el constructo de Inteligencia Emocional y el Rendimiento Académico en población universitaria. Tras los trabajos encontrados, hemos comprobado como ambas variables presentan una correlación estadísticamente significativa en todas las dimensiones que abarca la inteligencia emocional. Encontramos, además, que parece haber diferencias entre facultades (Páez \& Castaño, 2015) por lo que sería interesante replicar el nombrado estudio analizando las diferencias exactas entre las distintas disciplinas en referencia al perfil de inteligencia emocional presente tal y como hicieron Rodríguez et al. (2020).

En torno a las limitaciones, es destacable que sólo se ha trabajado en un tramo de 10 años y con tres bases de datos. Es posible que un análisis más profundo de esta relación saque a la luz novedosos aspectos clave que abran nuevas cuestiones de debate, por lo que se sugiere el uso de otras bases de datos y el estudio más prolongado en el tiempo, para vislumbrar esa relación desde una perspectiva histórica. También, se propone que se realice una nueva revisión sistemática que contemplen más idiomas además el castellano. Debido al auge de las publicaciones en inglés, se optó por una revisión en castellano que diera relevancia al idioma, pero sería de interés comprobar si las conclusiones de este estudio son iguales a otra hipotética revisión sistemática que usase bases de datos inglesas importantes como Scopus o Web of Science. Además, se propone el uso de bases de datos de menos relevancia, debido a que ignoramos donde podríamos encontrar un estudio revelador de alguna cuestión interesante. Por último, se resalta la importancia de seguir analizando esta relación, así como las múltiples variables y condiciones en las que puede ser estudiada. 


\section{Conclusión}

Los estudios que componen esta revisión concluyen, de forma unánime, la existencia de una relación directa significativa entre inteligencia emocional y rendimiento académico. Por tanto, altos niveles de Inteligencia Emocional predicen un nivel medio-alto académico.

\section{Referencias bibliográficas}

Acosta, F. P., \& Clavero, F. (2017). La inteligencia emocional como predictora del. Revista Complutense de Educación, 28(4), 1251-1259.

Apaza, M. (2018). La inteligencia emocional y su influencia en el rendimiento laboral en los trabajadores del Gobierno Reguional de Puno. Tesis doctoral, Universidad Nacional de Altiplano.

Buenrostro-Guerrero, A., Valadez-Sierra, M., Soltero-Avelar, R., Nava, G., Zambrano, R., \& García, A. (2012). Inteligencia emocional y rendimiento académico en adolescentes. Revista de educación y Desarrollo," 20(1), 29-37.

Caruso, D., \& Salovey, P. (2005). El directivo emocionalmente inteligente. La inteligencia emocional en la empresa. Málaga. España: Algaba.

Cejudo, J., López-Delgado, M. L., \& Rubio, M. J. (2016). Inteligencia emocional y resiliencia: su influencia en la satisfacción con la vida en estudiantes universitarios. Anuario de Psicología, 46(2), 51-57.

Del Rosal, I., Moreno-Manso, J. M., \& Bermejo, M. L. (2016). Inteligencia emocional y rendimiento académico en futuros maestros de la Universidad de Extremadura. Profesorado, 22(1), 257-275.

Díaz, I. C. (2013). Inteligencia emocional y rendimiento académico en estudiantes universitarios de Farmacia y Bioquímica en Huancayo. Apuntes de Ciencia y, 3(1), 36-50.

Fernández-Berrocal, P., \& Ruiz-Aranda, D. (2008). La inteligencia emocional en la educación. Revista Electrónica de Investigación Psicoeducativa, 6(2), 421-436.

Gámez-Medina, M. E., Guzmán-Facundo, F. R., Ahumada-Cortez, J. G., Alonso-Castillo, M. M., \& Da Silva Gherardi-Donato, E. C. (2017). Autoestima y consumo de alcohol en adolescentes escolarizados. NURE Investigación, 1-9.

Ilaja, B., \& Reyes, C. (2016). Burnout y estrategias de inteligencia emocional en profesores universitarios: implicaciones en la salud laboral educativa. Psicología desde el, 33(1), 31-46.

Jiménez, M., \& López-Zafra, E. (2013). Impacto de la inteligencia emocional percibida, actitudes sociales y expectativas del profesor en el rendimiento académico. Electronic, 11(1), 75-98. doi:https://www.redalyc.org/pdf/2931/293125761004.pdf

Luque-Reca, O. (2015). El papel de la inteligencia emocional sobre la calidad de vida en mayores institucionalizados. Jaen: Tesis doctoral, Universidad de Jaen.

Marín, M., Infante, E., \& Troyano, Y. (2000). El fracaso académico en la universidad: aspectos, motivaciones e intereses profesionales. revista Latinoamericana de Psicología, 32(3), 505-517.

Moreno, C. M., Vicente, E., \& Martínez, C. (1998). Revisión histórica del concepto de inteligencia: una aproximación a la inteligencia emocional. Revista, 30(1), 11-30. 
Núñez, A. (2018). Desarrollo de la Inteligencia Emocional en el alumnado con autismo. Revista de Investigación y Educación en Ciencias de la Salud, 3(2), 20-32.

Páez, M. L., \& Castaño, L. (2015). Inteligencia emocional y rendimiento académico en estudiantes universitarios. Psicología desde el Caribe, 32(2), 268-285.

Perestelo-Pérez, L. (2013). Standards on how to develop and report systematic reviews in Psychology and Health. International Journal of Clinical and Health Psychology, 13(1), 49-57.

Pérez, N., \& Castejón, J. (2007). La inteligencia emocional como predictor del rendimiento académico en estudiantes universitarios. Ansiedad y estrés, 13(1), 121-131.

Rodas, J., \& Rojas, M. (2015). El rendimiento académico y los niveles de inteligencia emocional. Revista de Educación y Cultura,, 4(1), 87-94.

Rodríguez, F. (2017). Relaciones entre afrontamiento del estrés cotidiano, autoconcepto, habilidades sociales e inteligencia emocional. European Journal of Education and Psychology, 10(2), 41-48.

Rodríguez, J., Rodríguez-Góngora, J., \& Rodríguez-Rodríguez, J. (2020). Inteligencia emocional en universitarios: análisis descriptivo en alumnos de 1으 y 2o de grado. Know and Share Psychology, 1(1), 23-37.

Salovey, P., \& Mayer, J. (1990). Emotional intelligence. Imagination, cognition and, 9(3), 185-211.

Sánchez-López, M. T., Megías, A., Gómez, R., Gutiérrez, M., \& Fernández-Berrocal, P. (2018). Relación entre la inteligencia emocional percibida y el comportamiento de riesgo en el ámbito de la salud. Escritos de Psicología, 11(3), 115-123.

Valenzuela-Santoyo, A., \& Portillo, S. (2018). La inteligencia emocional en educación primaria y su relación con el rendimiento académico. Revista Electrónica Educare, 22(3), 228-242.

Esta obra está bajo una Licencia Creative Commons Attribución-NoCommercial 4.0 International

(cc) BY-NC 